Short report

\title{
Proximal myopathy due to discrete carcinomatous metastases in muscle
}

\author{
R DOSHI, T FOWLER \\ From the Regional Neurological Unit, The Brook General Hospital, Woolwich, London, UK
}

SUMMARY Two patients are described with proximal muscle weakness and electromyographic evidence of a myopathic process. In both, discrete carcinomatous metastatic deposits were found in proximal muscles. The possible clinical implications are discussed.

Metastatic tumour deposits within skeletal muscle are uncommon. In a survey of necropsies in 500 cancer patients such deposits were found in only four patients. ${ }^{1}$ A detailed histological study of skeletal muscle in a routine necropsy survey found metastatic tumour nodules in $16 \%$ of 38 patients with malignant diseases. ${ }^{2}$ This was not due to direct invasion of muscle from local tumour. In a number of patients with malignant disease, skeletal muscle may show either inflammatory or degenerative changes, sometimes both. ${ }^{2}$ Clinical weakness may be explained in a number of ways. These include the non-metastatic carcinomatous neuromyopathies as well as myasthenic states, malignant cachexia, polymyositis and myopathies produced by ectopic hormone production.

Two patients are described who presented with proximal muscle weakness. Both had electromyographic (EMG) findings which supported a myopathic process, and one had added neuropathic features. In both the diagnosis of a non-metastatic carcinomatous neuromyopathy was considered although in both there were features to suggest the presence of more widespread carcinomatous involvement. In the first patient metastatic tumour deposits from a primary bronchogenic carcinoma were found in skeletal muscle at necropsy. In the second patient metastatic deposits from a primary breast carcinoma were found in a biopsy from the triceps muscle.

Address for reprint requests: Dr T Fowler, The Brook General Hospital, Shooters Hill Rd, Woolwich, London SE18 4LW, UK.

Received 28 July 1982 and in revised form 10 November 1982. Accepted 26 November 1982

\section{Case Histories}

Case 1 BM (195475) a 69-year-old housewife presented with a two month history of increasing weakness, malaise and weight loss. On admission she showed marked proximal muscle weakness and could only walk supported. She could not lift her arms above her head. The reflexes were preserved and the plantar responses flexor. Sensation was impaired in her feet. Over the next five weeks she deteriorated with increasing weakness and a terminal bronchopneumonia. Her chest radiograph showed left hilar enlargement and a carcinomatous neuromyopathy was suspected.

Investigations showed a haemoglobin of $11.2 \mathrm{~g} / \mathrm{dl}$ with ESR of $89 \mathrm{~mm} / \mathrm{h}$. Her liver function tests were abnormal and the serum proteins showed a high IgG level with compact band but a normal marrow biopsy. Normal values were found for her urea, electrolytes, creatine kinase (CK), calcium, thyroid function, auto-antibodies, DNA binding, skeletal survey and Tensilon test. An electroencephalogram showed a diffuse slow wave abnormality and a CT brain scan showed some patchy cerebral and cerebellar atrophy. EMG studies showed normal conduction but diminution in the amplitude of the sensory and nerve action potentials. In the right deltoid muscle myopathic potentials were found. No myasthenic features were elicited in the hypothenar muscles after exercise or repetitive stimulation. A left triceps muscle biopsy showed a few necrotic, rounded, highly eosinophilic fibres and some atrophic angular fibres. It was thought that these changes could be explained by a carcinomatous neuromyopathy. At necropsy she was found to have a carcinoma of the bronchus with local lymph gland involvement. Histologically the tumour was a poorly differentiated squamous cell carcinoma. There was no evidence of generalised metastatic spread. The triceps and quadriceps muscles showed fibre atrophy with deeply eosinophilic necrotic fibres surrounded by scanty inflammatory cell infiltrates. In addition clumps of malignant cells of squamous cell type were found between the muscle bundles and in the intermyofibrillary network (Fig. a). 

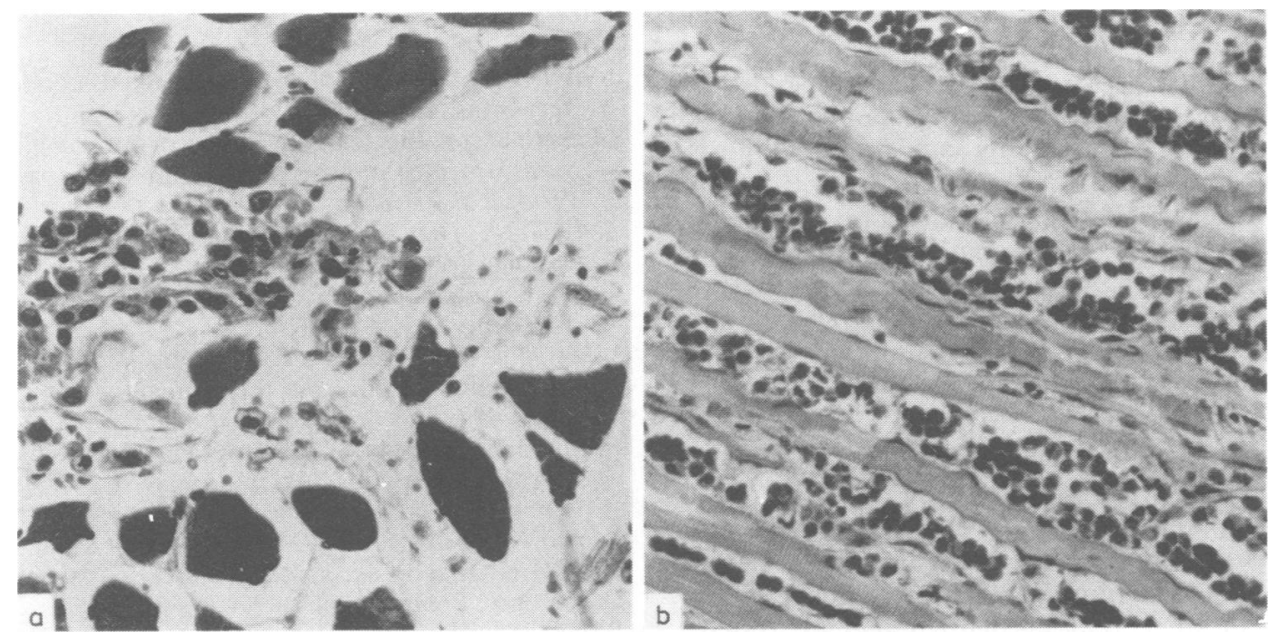

Fig (a) Group of large cells from a metastatic carcinoma lying in between the muscle fibres.

$H \& E \times 90$. (b) Clumps of small cells from a mucus secreting metastatic carcinoma in the intermyofibrillary network. $H \& E \times 75$.

Case 2 TM (35215) a 74-year-old widow presented with a three week history of increasing weakness with difficulty in walking. Six months before admission she developed easy bruising and anaemia and a marrow biopsy showed an amegakaryocytic thrombocytopenia. This responded to repeated transfusions and a course of steroids. Two months before the onset of weakness a left breast mass with axillary nodes were discovered and a local mastectomy was performed. The histology showed a scirrhous carcinoma. Within six weeks skin nodules had appeared. On admission she had evidence of skin secondaries on the chest wall. She could only walk supported and there was weakness and wasting of the proximal limb muscles and the neck flexors. The ankle jerks were depressed and vibration sense lost in the feet. It was thought she had carcinomatosis with a myopathy, the last probably arising as a non-metastatic complication.

Investigations confirmed she was anaemic with a haemoglobin of $8.3 \mathrm{~g} / \mathrm{dl}$, the white cell count was $12.5 \times$ $10 \%$ ( $28 \%$ polymorphs, $59 \%$ lymphocytes), the platelets normal, and the ESR $14 \mathrm{~mm} / 1 \mathrm{~h}$. The liver function tests were abnormal. Normal values were found for the serum. proteins; electrolytes, calcium, CK, cerebrospinal fluid and CT brain scan. A skeletal survey showed patchy sclerosis in both sides of the pelvis and in many ribs. EMG studies of the right deltoid and triceps muscles suggested a myopathic process. Nerve conduction studies showed no evidence of a generalised neuropathy. A left triceps muscle biopsy showed carcinomatous infiltration of the intermyofibrillary network by small round cells containing regular round nuclei with mucinous cytoplasm (fig b). Histochemical reactions revealed a uniform distribution of type 1 and 2 fibres but the cytoplasm of occasional fibres was penetrated by malignant cells, leaving the sarcolemmal sheath intact.

The patient was treated with tamoxifen with rapid improvement in muscle strength and resolution of the skin nodules. Twelve months after her presentation she was fully ambulant without obvious tumour recurrence and with only minimal proximal muscle weakness.

\section{Discussion}

In these two patients carcinomatosis was suspected and clinically proximal muscle weakness was present which was assumed to be caused by a non-metastatic neuromyopathy. Some possible neuropathic features were also found. The EMG studies supported a myopathic process and in the first patient there were some added neuropathic features.

Both carcinomatous infiltration of muscle by metastic deposits and muscle weakness in malignant diseases are well recognised although the clinical link does not appear to have been discussed widely. Local invasion of muscle from direct tumour spread is clearly described, but discrete metastatic deposits are far less frequent. Such deposits most commonly arise from rapidly growing anaplastic tumours. ${ }^{13}$ In an extensive survey of muscles examined from 110 routine necropsies, abnormalities were found in $49 \%$. These changes were most usually inflammatory or degenerative but six of 38 patients with malignant disease showed evidence of metastatic muscle deposits. ${ }^{2}$ There was no correlation between these findings and any specific muscle weakness. Metastatic deposits in muscle have also been found in the diaphragm, ${ }^{4}$ tongue, ${ }^{5}$ and extra-ocular muscles. ${ }^{6}$

Although muscle weakness and wasting are common in malignant disease non-metastatic neuromyopathic complications are rare. Such com- 
plications are said to have an incidence of $7 \%{ }^{7}$ and to be most common in lung cancer. EMG evidence of a myopathic process is most often found in those patients with polymyositis. In muscle biopsies from 24 cancer patients presenting with proximal muscle weakness, no metastatic deposits were found. ${ }^{8}$ Reports of EMG changes suggesting myopathic and neuropathic disturbances in patients with carcinoma are uncommon..$^{9-11}$ However lung cancer has the highest incidence and in one series approximately half of the patients had clinical evidence of proximal muscle weakness and one third depressed tendon reflexes. ${ }^{12}$ These were attributed to myopathy, neuromyopathy or a myopathic-myasthenic syndrome.

The two patients in this paper had discrete metastatic deposits in skeletal muscle which may well have contributed to the muscle weakness. Thus it is possible that in some patients with malignant disease, who show signs of muscle weakness, there may be discrete metastatic deposits in the muscles. However polymyositis, carcinomatous neuromyopathy or myopathic-myasthenic syndrome are more likely causes.

We are indebted to Dr D Barrett and Mr G NeilDwyer under whose care the second patient was admitted.

\section{References}

1 Willis RA. Pathology of Tumours. 4th ed. London: Butterworths, 1967.

${ }^{2}$ Pearson CM. Incidence and type of pathologic alterations observed in muscle in a routine autopsy survey. Neurology (Minneap) 1959;9:757-66.

${ }^{3}$ Willis RA. The Spread of Tumours in the Human Body. 3rd ed. London: Butterworths, 1973.

${ }^{4}$ Brennan JL. Metastatic tumours of the diaphragm. Br J Surg 1971;58:458-60.

s Zegarelli DJ, Tsukada Y, Pickren JW, Greene GW. Metastatic tumor to the tongue. Oral surgery 1973;35:202-11.

- Ashton N, Morgan G. Discrete carcinomatous metastases in the extraocular muscles. $\mathrm{Br} J$ Ophthalmol 1974;58:112-17.

${ }^{7}$ Croft PB, Wilkinson M. The incidence of carcinomatous neuromyopathy in patients with various types of carcinoma. Brain 1965;88:427-34.

${ }^{8}$ Shy GM. The late onset myopathy. World Neurology 1962;3:149-58.

9 Shy GM, Silverstein I. A study of the effects upon the motor unit by remote malignancy. Brain 1965;88:515-28.

${ }^{10}$ Moody JF. Electrophysiological investigations into the neurological complications of carcinoma. Brain 1965;88:1023-36.

"Trojaborg W, Frantzen E, Andersen I. Peripheral neuropathy and myopathy associated with carcinoma of the lung. Brain 1969;92:71-82.

${ }^{12}$ Campbell MJ, Paty DW. Carcinomatous neuromyopathy: 1. Electrophysiological studies. J Neurol Neurosurg Psychiatry 1974;37:131-41. 